\title{
A Note on the Rate of Strong Convergence for Weighted Sums of Arrays of Rowwise Negatively Orthant Dependent Random Variables
}

\author{
Qingxia Zhang and Dingcheng Wang \\ School of Mathematical Sciences, University of Electronic Science and Technology of China, Chengdu 611731, China \\ Correspondence should be addressed to Qingxia Zhang; zqx121981@126.com
}

Received 24 April 2014; Accepted 3 August 2014; Published 20 August 2014

Academic Editor: Baodong Zheng

Copyright (C) 2014 Q. Zhang and D. Wang. This is an open access article distributed under the Creative Commons Attribution License, which permits unrestricted use, distribution, and reproduction in any medium, provided the original work is properly cited.

Let $\left\{X_{n i} ; i \geq 1, n \geq 1\right\}$ be an array of rowwise negatively orthant dependent (NOD) random variables. The authors discuss the rate of strong convergence for weighted sums of arrays of rowwise NOD random variables and solve an open problem posed by Huang and Wang (2012).

\section{Introduction}

Firstly, let us recall the definitions of negatively associated (NA) random variables and NOD random variables as follows.

Definition 1. A finite collection of random variables $\left\{X_{i} ; 1 \leq\right.$ $i \leq n\}$ is said to be NA if for every pair of disjoint subsets $A_{1}$ and $A_{2}$ of $\{1,2, \ldots, n\}$,

$$
\operatorname{Cov}\left(f_{1}\left(X_{i}, i \in A_{1}\right), f_{2}\left(X_{j}, j \in A_{2}\right)\right) \leq 0
$$

whenever $f_{1}$ and $f_{2}$ are nondecreasing functions such that the covariance exists. An infinite collection of random variables $\left\{X_{i} ; i \geq 1\right\}$ is NA if every finite subcollection is NA.

An array of random variables $\left\{X_{n i} ; i \geq 1, n \geq 1\right\}$ is called rowwise NA random variables if for every $n \geq 1,\left\{X_{n i} ; i \geq 1\right\}$ is a sequence of NA random variables.
Definition 2. A finite collection of random variables $\left\{X_{i} ; 1 \leq\right.$ $i \leq n\}$ is said to be NOD if

$$
\begin{aligned}
& P\left(X_{1} \leq x_{1}, X_{2} \leq x_{2}, \ldots, X_{n} \leq x_{n}\right) \leq \prod_{j=1}^{n} P\left(X_{j} \leq x_{j}\right), \\
& P\left(X_{1}>x_{1}, X_{2}>x_{2}, \ldots, X_{n}>x_{n}\right) \leq \prod_{j=1}^{n} P\left(X_{j}>x_{j}\right),
\end{aligned}
$$

for all $x_{1}, x_{2}, \ldots, x_{n} \in \mathbb{R}$. An infinite collection of random variables $\left\{X_{i} ; i \geq 1\right\}$ is said to be NOD if every finite subcollection is NOD.

An array of random variables $\left\{X_{n i} ; i \geq 1, n \geq 1\right\}$ is called rowwise NOD random variables if for every $n \geq 1,\left\{X_{n i} ; i \geq 1\right\}$ is a sequence of NOD random variables.

The concepts of NA and NOD random variables were introduced by Joag-Dev and Proschan [1]. Obviously, independent random variables are NOD, and NA implies NOD from the definition of NA and NOD, but NOD does not imply NA. So, NOD is much weaker than NA. Because of the wide applications of NOD random variables, the notion of NOD random variables has been received more and more 
attention recently. Many applications have been found. We can refer to Volodin [2], Asadian et al. [3], Amini et al. [4, 5], Kuczmaszewska [6], Zarei and Jabbari [7], Wu and Zhu [8], $\mathrm{Wu}$ [9], Sung [10], Wang et al. [11], Huang and Wang [12], and so forth. Hence, it is very significant to study limit properties of this wider NOD random variables in probability theory and practical applications.

Let $\left\{X_{n} ; n \geq 1\right\}$ be a sequence of independent and identically distributed (i.i.d.) random variables and let $\left\{a_{n i} ; i \geq\right.$ $1, n \geq 1\}$ be an array of real constants. As Bai and Cheng [13] remarked, many useful linear statistics, for example, least-squares estimators, nonparametric regression function estimators, and jackknife estimates, are based on weighted sums of i.i.d. random variables. In this respect, the strong convergence for weighted sums $\sum_{i=1}^{n} a_{n i} X_{i}$ has been studied by many authors (see, e.g., Bai and Cheng [13]; Cuzick [14]; Sung [15]; Tang [16]; etc.).

Cai [17] proved the following complete convergence result for weighted sums of NA random variables.

Theorem A. Let $\left\{X, X_{n} ; n \geq 1\right\}$ be a sequence of identically distributed NA random variables, and let $\left\{a_{n i} ; 1 \leq i \leq n, n \geq\right.$ $1\}$ be an array of real constants satisfying

$$
A_{\alpha}=\limsup _{n \longrightarrow \infty} A_{\alpha, n}<\infty, \quad A_{\alpha, n}=\frac{1}{n} \sum_{i=1}^{n}\left|a_{n i}\right|^{\alpha},
$$

for some $0<\alpha \leq 2$. Suppose that $E X=0$ when $1<\alpha \leq 2$. If

$$
E\left\{\exp \left(h|X|^{\gamma}\right)\right\}<\infty \text { for some } h>0, \gamma>0 \text {, }
$$

then, for $b_{n}=n^{1 / \alpha}(\log n)^{1 / \gamma}$,

$$
\sum_{n=1}^{\infty} \frac{1}{n} P\left(\max _{1 \leq j \leq n}\left|\sum_{i=1}^{j} a_{n i} X_{i}\right|>\varepsilon b_{n}\right)<\infty \quad \forall \varepsilon>0 .
$$

Wang et al. [11] extended the above result of Cai [17] to arrays of rowwise NOD random variables as follows.

Theorem B. Let $\left\{X_{n i} ; i \geq 1, n \geq 1\right\}$ be an array of rowwise NOD random variables which is stochastically dominated by a random variable $X$ and let $\left\{a_{n i} ; 1 \leq i \leq n, n \geq 1\right\}$ be an array of real constants. Assume that there exist some $\delta$ with $0<\delta<1$ and some $\alpha$ with $0<\alpha<2$ such that $\sum_{i=1}^{n}\left|a_{n i}\right|^{\alpha}=O\left(n^{\delta}\right)$ and assume further that $E X_{n i}=0$ if $1<\alpha<2$. If for some $h>0$ and $\gamma>0$ such that (4), then

$$
\sum_{n=1}^{\infty} n^{\alpha p-2} P\left(\max _{1 \leq j \leq n}\left|\sum_{i=1}^{j} a_{n i} X_{n i}\right|>\varepsilon b_{n}\right)<\infty \quad \forall \varepsilon>0
$$

where $p \geq 1 / \alpha$ and $b_{n}=n^{1 / \alpha}(\log n)^{1 / \gamma}$.

Recently, Huang and Wang [12] partially extended the corresponding theorems of Cai [17] and Wang et al. [11] to NOD random variables under a mild moment condition.

Theorem C. Let $\left\{X_{n} ; n \geq 1\right\}$ be a sequence of NOD random variables which is stochastically dominated by a random variable $X$ and let $\left\{a_{n i} ; i \geq 1, n \geq 1\right\}$ be a triangular array of real constants such that $a_{n i}=0$ for $i>n$. Let

$$
A_{\beta}=\limsup _{n \longrightarrow \infty} A_{\beta, n}<\infty ; \quad A_{\beta, n}=n^{-1} \sum_{i=1}^{n}\left|a_{n i}\right|^{\beta} \text {, }
$$

where $\beta=\max (\alpha, \gamma)$ for some $0<\alpha \leq 2, \gamma>0$, and $\alpha \neq \gamma$. Assume that $E X_{n}=0$ for $1<\alpha \leq 2$ and $E|X|^{\beta}<\infty$. Then,

$$
\sum_{n=1}^{\infty} \frac{1}{n} P\left(\left|\sum_{i=1}^{n} a_{n i} X_{i}\right|>\varepsilon b_{n}\right)<\infty
$$

where $b_{n}=n^{1 / \alpha}(\log n)^{1 / \gamma}$.

As Huang and Wang [12] pointed out, Theorem C partially extends only the case of $\alpha>\gamma$ of Theorems A and B. They left an open problem whether the case of $\alpha=\gamma$ of Theorem $\mathrm{C}$ holds for NOD random variables.

The main purpose of this paper is to further study strong convergence for weighted sums of NOD random variables and to obtain the rate of strong convergence for weighted sums of arrays of rowwise NOD random variables under a suitable moment condition. We solve the above problem posed by Huang and Wang [12].

We will use the following concept in this paper.

Definition 3. An array of random variables $\left\{X_{n i} ; i \geq 1, n \geq 1\right\}$ is said to be stochastically dominated by a random variable $X$ if there exists a positive constant $C$ such that

$$
P\left(\left|X_{n i}\right|>t\right) \leq C P(|X|>t),
$$

for all $t \geq 0, i \geq 1$, and $n \geq 1$.

\section{Main Results}

Now, we will present the main results of this paper; the detailed proofs will be given in the next section.

Theorem 4. Let $\left\{X_{n i} ; i \geq 1, n \geq 1\right\}$ be an array of rowwise NOD random variables which is stochastically dominated by a random variable $X$ and let $\left\{a_{n i} ; 1 \leq i \leq n, n \geq 1\right\}$ be an array of real constants satisfying $\sum_{i=1}^{n}\left|a_{n i}\right|^{\alpha}=O(n)$ for some $0<\alpha \leq 2$. Assume further that $E X_{n i}=0$ for $1<\alpha \leq 2$ and $E|X|^{\alpha} \log (1+|X|)<\infty$. Then,

$$
\sum_{n=1}^{\infty} n^{-1} P\left(\left|\sum_{i=1}^{n} a_{n i} X_{n i}\right|>\varepsilon b_{n}\right)<\infty \quad \forall \varepsilon>0,
$$

where $b_{n}=n^{1 / \alpha}(\log n)^{1 / \alpha}$.

Similar to the proof of Theorem 4, we can obtain the following result for NOD random variable sequences.

Corollary 5. Let $\left\{X_{n} ; n \geq 1\right\}$ be a sequence of NOD random variables which is stochastically dominated by a random variable $X$ and let $\left\{a_{n i} ; 1 \leq i \leq n, n \geq 1\right\}$ be an array of real constants satisfying $\sum_{i=1}^{n}\left|a_{n i}\right|^{\alpha}=O(n)$ for some 
$0<\alpha \leq 2$. Assume further that $E X_{n}=0$ for $1<\alpha \leq 2$ and $E|X|^{\alpha} \log (1+|X|)<\infty$. Then,

$$
\sum_{n=1}^{\infty} n^{-1} P\left(\left|\sum_{i=1}^{n} a_{n i} X_{i}\right|>\varepsilon b_{n}\right)<\infty \quad \forall \varepsilon>0,
$$

where $b_{n}=n^{1 / \alpha}(\log n)^{1 / \alpha}$.

Remark 6. In Theorem 4 and Corollary 5, we consider the case of $\alpha=\gamma$ for $0<\alpha \leq 2$ and obtain some strong convergence results for arrays of rowwise NOD random variables and NOD random variable sequences without assumption of identical distribution. The main result settles the open problem posed by Huang and Wang [12]. In addition, it is still an open problem whether

$$
\sum_{n=1}^{\infty} n^{-1} P\left(\max _{1 \leq j \leq n}\left|\sum_{i=1}^{j} a_{n i} X_{n i}\right|>\varepsilon b_{n}\right)<\infty \quad \forall \varepsilon>0
$$

holds true under the same moment condition of Theorem 4.

\section{Proofs}

In order to prove our main results, the following lemmas are needed.

Lemma 7 (see Bozorgnia et al. [18]). Let $\left\{X_{i} ; 1 \leq i \leq n\right\}$ be a sequence of NOD random variables, and let $\left\{f_{i} ; 1 \leq\right.$ $i \leq n\}$ be a sequence of Borel functions all of which are monotone nondecreasing (or all are monotone nonincreasing). Then, $\left\{f_{i}\left(X_{i}\right) ; 1 \leq i \leq n\right\}$ is a sequence of NOD random variables.

Lemma 8 (see Asadian et al. [3]). Let $M \geq 2$ and let $\left\{X_{n} ; n \geq\right.$ $1\}$ be a sequence of NOD random variables with $E X_{n}=0$ and $E\left|X_{n}\right|^{M}<\infty$ for all $n \geq 1$. Then, there exists a positive constant $C=C(M)$ depending only on $M$ such that, for all $n \geq 1$,

$$
E\left(\left|\sum_{i=1}^{n} X_{i}\right|^{M}\right) \leq C\left[\sum_{i=1}^{n} E\left|X_{i}\right|^{M}+\left(\sum_{i=1}^{n} E X_{i}^{2}\right)^{M / 2}\right] .
$$

Lemma 9. Let $\left\{X_{n} ; n \geq 1\right\}$ be a sequence of random variables which is stochastically dominated by a random variable $X$. For any $u>0$ and $t>0$, the following two statements hold:

$$
E\left|X_{n i}\right|^{u} I\left(\left|X_{n i}\right| \leq t\right) \leq C_{1}\left(E|X|^{u} I(|X| \leq t)+t^{u} P(|X|>t)\right),
$$

$$
E\left|X_{n i}\right|^{u} I\left(\left|X_{n i}\right|>t\right) \leq C_{2} E|X|^{u} I(|X|>t)
$$

where $C_{1}$ and $C_{2}$ are positive constants.

Lemma 10 (see Sung [15]). Let $X$ be a random variable and let $\left\{a_{n i} ; 1 \leq i \leq n, n \geq 1\right\}$ be an array of real constants satisfying $\sum_{i=1}^{n}\left|a_{n i}\right|^{\alpha}=O(n)$ for some $\alpha>0$. Let $b_{n}=n^{1 / \alpha}(\log n)^{1 / \gamma}$ for some $\gamma>0$. Then,

$$
\begin{aligned}
& \sum_{n=1}^{\infty} n^{-1} \sum_{i=1}^{n} P\left(\left|a_{n i} X\right|>b_{n}\right) \text { for } \alpha>\gamma, \\
& \quad \leq \begin{cases}C E|X|^{\alpha}, & \text { for } \alpha=\gamma, \\
C E|X|^{\alpha} \log (1+|X|), & \text { for } \alpha<\gamma . \\
C E|X|^{\gamma}, & \text { f }\end{cases}
\end{aligned}
$$

Lemma 11 (see Sung [19]). Let $X$ be a random variable and let $\left\{a_{n i} ; 1 \leq i \leq n, n \geq 1\right\}$ be an array of real constants satisfying $a_{n i}=0$ or $\left|a_{n i}\right|>1$ and $\sum_{i=1}^{n}\left|a_{n i}\right|^{\alpha}=O(n)$ for some $\alpha>0$. Let $b_{n}=n^{1 / \alpha}(\log n)^{1 / \alpha}$. If $q>\alpha$, then

$$
\begin{aligned}
& \sum_{n=1}^{\infty} n^{-1} b_{n}^{-q} \sum_{i=1}^{n} E\left|a_{n i} X\right|^{q} I\left(\left|a_{n i} X\right| \leq b_{n}\right) \\
& \leq C E|X|^{\alpha} \log (1+|X|) .
\end{aligned}
$$

Throughout this paper, let $I(A)$ be the indicator function of the set $A$. $C$ denotes a positive constant, which may be different in various places and $a_{n}=O\left(b_{n}\right)$ stands for $a_{n} \leq C b_{n}$.

Proof of Theorem 4. Without loss of generality, suppose that $\sum_{i=1}^{n}\left|a_{n i}\right|^{\alpha} \leq C n$ and $a_{n i} \geq 0$, for all $1 \leq i \leq n, n \geq 1$. For fixed $n \geq 1$, define

$$
\begin{gathered}
X_{i}^{(n)}=-b_{n} I\left(a_{n i} X_{n i}<-b_{n}\right)+a_{n i} X_{n i} I\left(\left|a_{n i} X_{n i}\right| \leq b_{n}\right) \\
+b_{n} I\left(a_{n i} X_{n i}>b_{n}\right), \quad i \geq 1, \\
T_{n}^{(n)}=\sum_{i=1}^{n}\left(X_{i}^{(n)}-E X_{i}^{(n)}\right) .
\end{gathered}
$$

Denote

$$
\begin{aligned}
A & =\bigcap_{i=1}^{n}\left(a_{n i} X_{n i}=X_{i}^{(n)}\right), \\
B & =\bar{A}=\bigcup_{i=1}^{n}\left(a_{n i} X_{n i} \neq X_{i}^{(n)}\right) \\
& =\bigcup_{i=1}^{n}\left(\left|a_{n i} X_{n i}\right|>b_{n}\right), \\
E_{n} & =\left(\left|\sum_{i=1}^{n} a_{n i} X_{n i}\right|>\varepsilon b_{n}\right) .
\end{aligned}
$$

It is easily seen that, for all $\varepsilon>0$,

$$
\begin{gathered}
E_{n}=E_{n} A \bigcup E_{n} B \subset\left(\left|\sum_{i=1}^{n} X_{i}^{(n)}\right|>\varepsilon b_{n}\right) \\
\bigcup\left(\bigcup_{i=1}^{n}\left|a_{n i} X_{n i}\right|>b_{n}\right),
\end{gathered}
$$


which implies that

$$
\begin{aligned}
P\left(E_{n}\right) & \leq P\left(\left|\sum_{i=1}^{n} X_{i}^{(n)}\right|>\varepsilon b_{n}\right)+P\left(\bigcup_{i=1}^{n}\left|a_{n i} X_{n i}\right|>b_{n}\right) \\
& \leq P\left(\left|T_{n}^{(n)}\right|>\varepsilon b_{n}-\left|\sum_{i=1}^{n} E X_{i}^{(n)}\right|\right)+\sum_{i=1}^{n} P\left(\left|a_{n i} X_{n i}\right|>b_{n}\right) .
\end{aligned}
$$

First, we will prove that

$$
b_{n}^{-1}\left|\sum_{i=1}^{n} E X_{i}^{(n)}\right| \longrightarrow 0, \quad \text { as } n \longrightarrow \infty .
$$

Actually, for $0<\alpha \leq 1$, by (14) of Lemma 9, Markov inequality, and $E|X|^{\alpha} \log (1+|X|)<\infty$, we have that

$$
\begin{aligned}
b_{n}^{-1}\left|\sum_{i=1}^{n} E X_{i}^{(n)}\right| \leq & C b_{n}^{-1} \sum_{i=1}^{n}\left|E X_{i}^{(n)}\right| \\
\leq & C b_{n}^{-1} \sum_{i=1}^{n} E\left|a_{n i} X_{n i}\right| I\left(\left|a_{n i} X_{n i}\right| \leq b_{n}\right) \\
& +C \sum_{i=1}^{n} P\left(\left|a_{n i} X_{n i}\right|>b_{n}\right) \\
\leq & C b_{n}^{-1} \sum_{i=1}^{n}\left(E\left|a_{n i} X\right| I\left(\left|a_{n i} X\right| \leq b_{n}\right)\right. \\
& +C \sum_{i=1}^{n} P\left(\left|a_{n i} X\right|>b_{n}\right) \\
\leq & C b_{n}^{-1} \sum_{i=1}^{n}\left(E\left|a_{n i} X\right| I\left(\left|a_{n i} X\right| \leq b_{n}\right)\right) \\
& +C \sum_{i=1}^{n} P\left(\left|a_{n i} X\right|>b_{n}\right) \\
\leq & C b_{n}^{-\alpha} \sum_{i=1}^{n}\left(E\left|a_{n i} X\right|^{\alpha} I\left(\left|a_{n i} X\right| \leq b_{n}\right)\right) \\
& \left.\left.+C b_{n}^{-\alpha} \sum_{i=1}^{n} E\left|a_{n i} X\right|^{\alpha} X \mid b_{n}\right)\right) \\
\leq & C b_{n}^{-\alpha} \sum_{i=1}^{n}\left|a_{n i}\right|^{\alpha} E|X|^{\alpha}+C b_{n}^{-\alpha} \sum_{i=1}^{n}\left|a_{n i}\right|^{\alpha} E|X|^{\alpha} E|X|^{\alpha} \longrightarrow 0 \quad \text { as } n \longrightarrow \infty .
\end{aligned}
$$

Next, for $1<\alpha \leq 2$, by $E X_{n i}=0$, (15) of Lemmas 9 and 10, Markov inequality, and $E|X|^{\alpha} \log (1+|X|)<\infty$, we also have that

$$
\begin{aligned}
b_{n}^{-1}\left|\sum_{i=1}^{n} E X_{i}^{(n)}\right| \leq & C \sum_{i=1}^{n} P\left(\left|a_{n i} X_{n i}\right|>b_{n}\right) \\
& +C b_{n}^{-1}\left|\sum_{i=1}^{n} E a_{n i} X_{n i} I\left(\left|a_{n i} X_{n i}\right|>b_{n}\right)\right| \\
\leq & C \sum_{i=1}^{n} P\left(\left|a_{n i} X\right|>b_{n}\right) \\
& +C b_{n}^{-1} \sum_{i=1}^{n} E\left|a_{n i} X X_{n i}\right| I\left(\left|a_{n i} X_{n i}\right|>b_{n}\right) \\
\leq & C b_{n}^{-\alpha} \sum_{i=1}^{n} E\left|a_{n i} X\right|^{\alpha} \\
& +C b_{n}^{-1} \sum_{i=1}^{n} E\left|a_{n i} X\right| I\left(\left|a_{n i} X\right|>b_{n}\right) \\
\leq & C b_{n}^{-\alpha} \sum_{i=1}^{n} E\left|a_{n i} X\right|^{\alpha} \\
& +C b_{n}^{-\alpha} \sum_{i=1}^{n} E\left|a_{n i} X\right|^{\alpha} I\left(\left|a_{n i} X\right|>b_{n}\right) \\
\leq & C b_{n}^{-\alpha} \sum_{i=1}^{n} E\left|a_{n i} X\right|^{\alpha}+C b_{n}^{-\alpha} \sum_{i=1}^{n} E\left|a_{n i} X\right|^{\alpha} \\
\leq & C(\log n)^{-1} E|X|^{\alpha} \longrightarrow 0 \quad \text { as } n \longrightarrow \infty .
\end{aligned}
$$

From the above statements, we can get (22) immediately. Hence, for $n$ large enough,

$$
P\left(E_{n}\right) \leq P\left(\left|T_{n}^{(n)}\right|>\frac{\varepsilon b_{n}}{2}\right) .
$$

To prove (10), it is sufficient to show that

$$
\begin{gathered}
I \triangleq \sum_{n=1}^{\infty} n^{-1} P\left(\left|T_{n}^{(n)}\right|>\frac{\varepsilon b_{n}}{2}\right)<\infty, \\
J \triangleq \sum_{n=1}^{\infty} n^{-1} \sum_{i=1}^{n} P\left(\left|a_{n i} X_{n i}\right|>b_{n}\right)<\infty .
\end{gathered}
$$

It follows from Lemma 10 and $E|X|^{\alpha} \log (1+|X|)<\infty$ that

$$
\begin{aligned}
J & \triangleq \sum_{n=1}^{\infty} n^{-1} \sum_{i=1}^{n} P\left(\left|a_{n i} X_{n i}\right|>b_{n}\right) \\
& \leq C \sum_{n=1}^{\infty} n^{-1} \sum_{i=1}^{n} P\left(\left|a_{n i} X\right|>b_{n}\right) \\
& \leq E|X|^{\alpha} \log (1+|X|)<\infty .
\end{aligned}
$$

For fixed $n \geq 1$, it is easily seen that $\left\{X_{i}^{(n)}-E X_{i}^{(n)}, i \geq 1, n \geq 1\right\}$ is still a sequence of NOD random variables with mean zero 
by Lemma 7. Hence, it follows from (14) of Lemmas 9 and 8 and Markov inequality (for $M>2$ ) that

$$
\begin{aligned}
I \triangleq & \sum_{n=1}^{\infty} n^{-1} P\left(\left|T_{n}^{(n)}\right|>\frac{\varepsilon b_{n}}{2}\right) \\
\leq & C \sum_{n=1}^{\infty} n^{-1} b_{n}^{-M} E\left(\left|T_{n}^{(n)}\right|^{M}\right) \\
\leq & C \sum_{n=1}^{\infty} n^{-1} b_{n}^{-M}\left[\sum_{i=1}^{n} E\left|X_{i}^{(n)}\right|^{M}+\left(\sum_{i=1}^{n} E\left|X_{i}^{(n)}\right|^{2}\right)^{M / 2}\right] \\
\leq & C \sum_{n=1}^{\infty} n^{-1} b_{n}^{-M} \sum_{i=1}^{n} E\left|X_{i}^{(n)}\right|^{M} \\
& +C \sum_{n=1}^{\infty} n^{-1} b_{n}^{-M}\left(\sum_{i=1}^{n} E\left|X_{i}^{(n)}\right|^{2}\right)^{M / 2} \\
\triangleq & I_{1}+I_{2} .
\end{aligned}
$$

It follows from Lemma 10, (14) of Lemma 9, and Markov inequality that

$$
\begin{aligned}
I_{1} & \triangleq C \sum_{n=1}^{\infty} n^{-1} b_{n}^{-M} \sum_{i=1}^{n} E\left|X_{i}^{(n)}\right|^{M} \\
\leq & C \sum_{n=1}^{\infty} n^{-1} b_{n}^{-M}\left\{\sum_{i=1}^{n}\left|a_{n i}\right|^{M} E\left|X_{n i}\right|^{M} I\left(\left|a_{n i} X_{n i}\right| \leq b_{n}\right)\right. \\
& \left.+\sum_{i=1}^{n} b_{n}^{M} P\left(\left|a_{n i} X_{n i}\right|>b_{n}\right)\right\} \\
\leq & C \sum_{n=1}^{\infty} n^{-1} b_{n}^{-M}\left\{\sum_{i=1}^{n}\left|a_{n i}\right|^{M} E|X|^{M} I\left(\left|a_{n i} X\right| \leq b_{n}\right)\right. \\
\leq & C \sum_{n=1}^{\infty} n^{-1} b_{n}^{-M} \sum_{i=1}^{n}\left|a_{n i}\right|^{M} E|X|^{M} I\left(\left|a_{n i} X\right| \leq b_{n}\right) \\
& \left.\quad+2 \sum_{i=1}^{n} b_{n}^{M} P\left(\left|a_{n i} X\right|>b_{n}\right)\right\} \\
& +C \sum_{n=1}^{\infty} n^{-1} \sum_{i=1}^{n} P\left(\left|a_{n i} X\right|>b_{n}\right) \\
& +I_{12} \cdot
\end{aligned}
$$

From Lemma 10 and $E|X|^{\alpha} \log (1+|X|)<\infty$, we can obtain that

$$
\begin{aligned}
I_{12} & \triangleq C \sum_{n=1}^{\infty} n^{-1} \sum_{i=1}^{n} P\left(\left|a_{n i} X\right|>b_{n}\right) \\
& \leq E|X|^{\alpha} \log (1+|X|)<\infty .
\end{aligned}
$$

For fixed $n>1$, we divide $\left\{a_{n i}, 1 \leq i \leq n\right\}$ into three subsets $\left\{a_{n i}:\left|a_{n i}\right| \leq 1 /(\log n)^{m}\right\},\left\{a_{n i}: 1 /(\log n)^{m}<\left|a_{n i}\right| \leq 1\right\}$, and $\left\{a_{n i}:\left|a_{n i}\right|>1\right\}$, where $m=(1 /(M-\alpha))$. Then,

$$
\begin{aligned}
I_{11} \triangleq & C \sum_{n=1}^{\infty} n^{-1} b_{n}^{-M} \sum_{i=1}^{n}\left|a_{n i}\right|^{M} E|X|^{M} I\left(\left|a_{n i} X\right| \leq b_{n}\right) \\
= & C \sum_{n=1}^{\infty} n^{-1} b_{n}^{-M} \\
& \times \sum_{i:\left|a_{n i}\right| \leq 1 /(\log n)^{m}}\left|a_{n i}\right|^{M} E|X|^{M} I\left(\left|a_{n i} X\right| \leq b_{n}\right) \\
& +C \sum_{n=1}^{\infty} n^{-1} b_{n}^{-M} \sum_{i: 1 /(\log n)^{m}<\left|a_{n i}\right| \leq 1}\left|a_{n i}\right|^{M} E|X|^{M} I\left(\left|a_{n i} X\right| \leq b_{n}\right) \\
& +C \sum_{n=1}^{\infty} n^{-1} b_{n}^{-M} \sum_{i:\left|a_{n i}\right|>1}\left|a_{n i}\right|^{M} E|X|^{M} I\left(\left|a_{n i} X\right| \leq b_{n}\right) \\
\triangleq & I_{11}^{(1)}+I_{11}^{(2)}+I_{11}^{(3)} .
\end{aligned}
$$

By Lemma 11 and $E|X|^{\alpha} \log (1+|X|)<\infty$ again, it follows that

$$
\begin{aligned}
I_{11}^{(3)} & \triangleq C \sum_{n=1}^{\infty} n^{-1} b_{n}^{-M} \sum_{i:\left|a_{n i}\right|>1}\left|a_{n i}\right|^{M} E|X|^{M} I\left(\left|a_{n i} X\right| \leq b_{n}\right) \\
& \leq E|X|^{\alpha} \log (1+|X|)<\infty \quad \text { for } M>2 \geq \alpha>0 .
\end{aligned}
$$

Noting that $\sum_{i:\left|a_{n i}\right| \leq 1 /(\log n)^{m}}\left|a_{n i}\right|^{\alpha} \leq C n(\log n)^{-m \alpha}$, for $M>\alpha$ and fixed $n>1$, we have that

$$
\begin{aligned}
I_{11}^{(1)} & \triangleq C \sum_{n=1}^{\infty} n^{-1} b_{n}^{-M} \sum_{i:\left|a_{n i}\right| \leq 1 /(\log n)^{m}}\left|a_{n i}\right|^{M} E|X|^{M} I\left(\left|a_{n i} X\right| \leq b_{n}\right) \\
& \leq C \sum_{n=1}^{\infty} n^{-1} b_{n}^{-\alpha} \sum_{i:\left|a_{n i}\right| \leq 1 /(\log n)^{m}}\left|a_{n i}\right|^{\alpha} E|X|^{\alpha} I\left(\left|a_{n i} X\right| \leq b_{n}\right) \\
& \leq C E|X|^{\alpha} \sum_{n=1}^{\infty} n^{-1} b_{n}^{-\alpha} \sum_{i:\left|a_{n i}\right| \leq 1 /(\log n)^{m}}\left|a_{n i}\right|^{\alpha} \\
& \leq C E|X|^{\alpha} \sum_{n=1}^{\infty} n^{-1} n^{-1}(\log n)^{-1} n(\log n)^{-m \alpha}<\infty .
\end{aligned}
$$


Noting that $\sum_{i: 1 /(\log n)^{m}<\left|a_{n i}\right| \leq 1}\left|a_{n i}\right|^{M} \leq C n$ and $m=1 /(M-\alpha)$, for $M>2,0<\alpha \leq 2$, we have that

$$
\begin{aligned}
I_{11}^{(2)} \triangleq & C \sum_{n=1}^{\infty} n^{-1} b_{n}^{-M} \sum_{i: 1 /(\log n)^{m}<\left|a_{n i}\right| \leq 1}\left|a_{n i}\right|^{M} E|X|^{M} I\left(\left|a_{n i} X\right| \leq b_{n}\right) \\
\leq & C \sum_{n=1}^{\infty} b_{n}^{-M} E|X|^{M} I\left(|X| \leq b_{n}(\log n)^{m}\right) \\
= & C \sum_{n=1}^{\infty} b_{n}^{-M} \sum_{k=1}^{n} E|X|^{M} I\left((k-1)^{1 / \alpha}(\log (k-1))^{m+1 / \alpha}\right. \\
= & C \sum_{k=1}^{\infty} E|X|^{M} I\left((k-1)^{1 / \alpha}(\log (k-1))^{m+1 / \alpha}\right. \\
& \times \sum_{n=k}^{\infty} n^{-M / \alpha}(\log n)^{-M / \alpha} \\
\leq & C \sum_{k=1}^{\infty} E|X|^{M} I\left((k-1)^{1 / \alpha}(\log (k-1))^{m+1 / \alpha}\right. \\
\leq & \left.\quad C E|X|^{\alpha}<\infty \sum_{k=1}^{m+1 / \alpha}\right) \\
& \left.\times k^{1 / \alpha}(\log k)^{m+1 / \alpha}\right) \\
<|X|^{\alpha} I\left((k-1)^{1 / \alpha}(\log (k-1))^{m+1 / \alpha}\right. & \left.<|X| \leq k^{1 / \alpha}(\log k)^{m+1 / \alpha}\right) \\
& <(\log k)^{-M / \alpha}
\end{aligned}
$$

Finally, we will prove that

$$
I_{2} \triangleq C \sum_{n=1}^{\infty} n^{-1} b_{n}^{-M}\left(\sum_{i=1}^{n} E\left|X_{i}^{(n)}\right|^{2}\right)^{M / 2}<\infty .
$$

Hence, by $C_{r}$ inequality, Markov inequality, Lemmas 9-11, and $E|X|^{\alpha} \log (1+|X|)<\infty$, we have that

$$
\begin{aligned}
I_{2} & \triangleq C \sum_{n=1}^{\infty} n^{-1} b_{n}^{-M}\left(\sum_{i=1}^{n} E\left|X_{i}^{(n)}\right|^{2}\right)^{M / 2} \\
& =C \sum_{n=1}^{\infty} n^{-1}\left(\sum_{i=1}^{n} b_{n}^{-2} E\left|X_{i}^{(n)}\right|^{2}\right)^{M / 2} \\
& \leq C \sum_{n=1}^{\infty} n^{-1}\left(\sum_{i=1}^{n} P\left(\left|a_{n i} X_{n i}\right|>b_{n}\right)\right)^{M / 2}
\end{aligned}
$$

$$
\begin{aligned}
& +C \sum_{n=1}^{\infty} n^{-1}\left(\sum_{i=1}^{n} b_{n}^{-2} E\left|a_{n i} X_{n i}\right|^{2}\left(\left|a_{n i} X_{n i}\right| \leq b_{n}\right)\right)^{M / 2} \\
\leq & C\left(\sum_{n=1}^{\infty} n^{-1} \sum_{i=1}^{n} P\left(\left|a_{n i} X\right|>b_{n}\right)\right)^{M / 2} \\
& +C\left(\sum_{n=1}^{\infty} n^{-1} \sum_{i=1}^{n} b_{n}^{-2} E\left|a_{n i} X\right|^{2}\left(\left|a_{n i} X\right| \leq b_{n}\right)\right)^{M / 2} \\
\leq & C\left(E|X|^{\alpha} \log (1+|X|)\right)^{M / 2}<\infty .
\end{aligned}
$$

Therefore, the desired result (10) follows from the above statements. This completes the proof of Theorem 4 .

\section{Conflict of Interests}

The authors declare that they have no conflict of interests.

\section{Acknowledgments}

The authors are most grateful to the referees and to the editor Professor Baodong Zheng for their valuable suggestions and some helpful comments which greatly improved the clarity and readability of this paper. This paper is partially supported by the National Nature Science Foundation of China (71271042), the Fundamental Research Funds for the Central Universities of China (ZYGX2012J119), the Nature Science Foundation of Guangxi Province (2014GXNSFBA118006, 2013GXNSFDA019001), and the Guangxi Provincial Scientific Research Projects (201204LX157, 2013YB104).

\section{References}

[1] K. Joag-Dev and F. Proschan, "Negative association of random variables, with applications," The Annals of Statistics, vol. 11, no. 1, pp. 286-295, 1983.

[2] A. Volodin, "On the Kolmogorov exponential inequality for negatively dependent random variables," Pakistan Journal of Statistics, vol. 18, no. 2, pp. 249-254, 2002.

[3] N. Asadian, V. Fakoor, and A. Bozorgnia, "Rosental's type inequalities for negatively orthant dependent random variables," Journal of Iranian Statistical Society, vol. 5, no. 1-2, pp. 66-75, 2006

[4] M. Amini and A. Bozorgnia, "Complete convergence for negatively dependent random variables," Journal of Applied Mathematics and Stochastic Analysis, vol. 16, no. 2, pp. 121-126, 2003.

[5] M. Amini, H. Zarei, and A. Bozorgnia, "Some strong limit theorems of weighted sums for negatively dependent generalized Gaussian random variables," Statistics \& Probability Letters, vol. 77, no. 11, pp. 1106-1110, 2007.

[6] A. Kuczmaszewska, "On some conditions for complete convergence for arrays of rowwise negatively dependent random variables," Stochastic Analysis and Applications, vol. 24, no. 6, pp. 1083-1095, 2006. 
[7] H. Zarei and H. Jabbari, "Complete convergence of weighted sums under negative dependence," Statistical Papers, vol. 52, no. 2, pp. 413-418, 2011.

[8] Y. Wu and D. Zhu, "Convergence properties of partial sums for arrays of rowwise negatively orthant dependent random variables," Journal of the Korean Statistical Society, vol. 39, no. 2, pp. 189-197, 2010.

[9] Q. Wu, "Complete convergence for negatively dependent sequences of random variables," Journal of Inequalities and Applications, vol. 2010, Article ID 507293, 10 pages, 2010.

[10] S. H. Sung, "A note on the complete convergence for arrays of dependent random variables," Journal of Inequalities and Applications, vol. 2011, article 76, 2011.

[11] X. Wang, S. Hu, and W. Yang, "Complete convergence for arrays of rowwise negatively orthant dependent random variables," RACSAM, vol. 106, no. 2, pp. 235-245, 2012.

[12] H. W. Huang and D. C. Wang, "A note on the strong limit theorem for weighted sums of sequences of negatively dependent random variables," Journal of Inequalities and Applications, vol. 2012, article 233, 2012.

[13] Z. D. Bai and P. E. Cheng, "Marcinkiewicz strong laws for linear statistics," Statistics \& Probability Letters, vol. 46, no. 2, pp. 105$112,2000$.

[14] J. Cuzick, "A strong law for weighted sums of i.i.d. random variables," Journal of Theoretical Probability, vol. 8, no. 3, pp. 625-641, 1995.

[15] S. H. Sung, "On the strong convergence for weighted sums of random variables," Statistical Papers, vol. 52, no. 2, pp. 447-454, 2011.

[16] X. Tang, "Strong convergence results for arrays of rowwise pairwise NQD random variables," Journal of Inequalities and Applications, vol. 2013, article 102, 2013.

[17] G. Cai, "Strong laws for weighted sums of NA random variables," Metrika, vol. 68, no. 3, pp. 323-331, 2008.

[18] A. Bozorgnia, R. F. Patterson, and R. L. Taylor, "Limit theorems for dependent random variables," in World Congress of Nonlinear Analysts '92, Vol. I-IV (Tampa, FL, 1992), pp. 1639-1650, de Gruyter, Berlin, Germany, 1996.

[19] S. H. Sung, "On the strong convergence for weighted sums of $\rho^{*}$ - mixing random variables," Statistical Papers, vol. 54, pp. 773-781, 2013. 


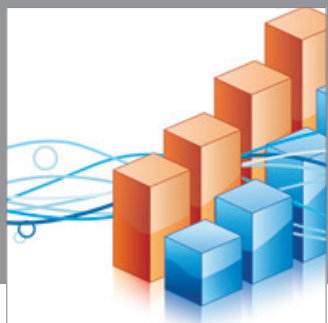

Advances in

Operations Research

mansans

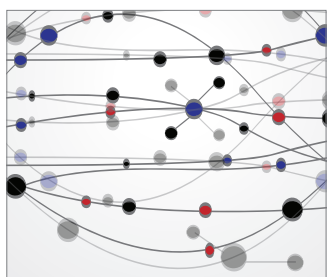

The Scientific World Journal
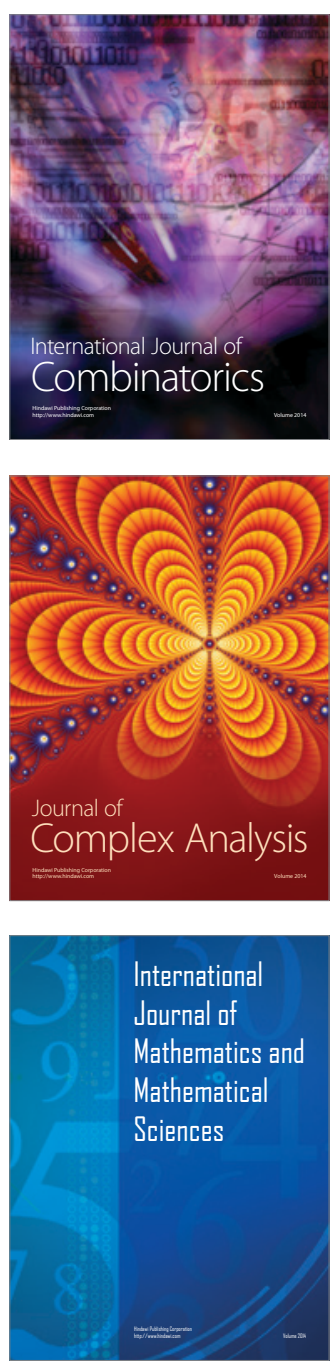
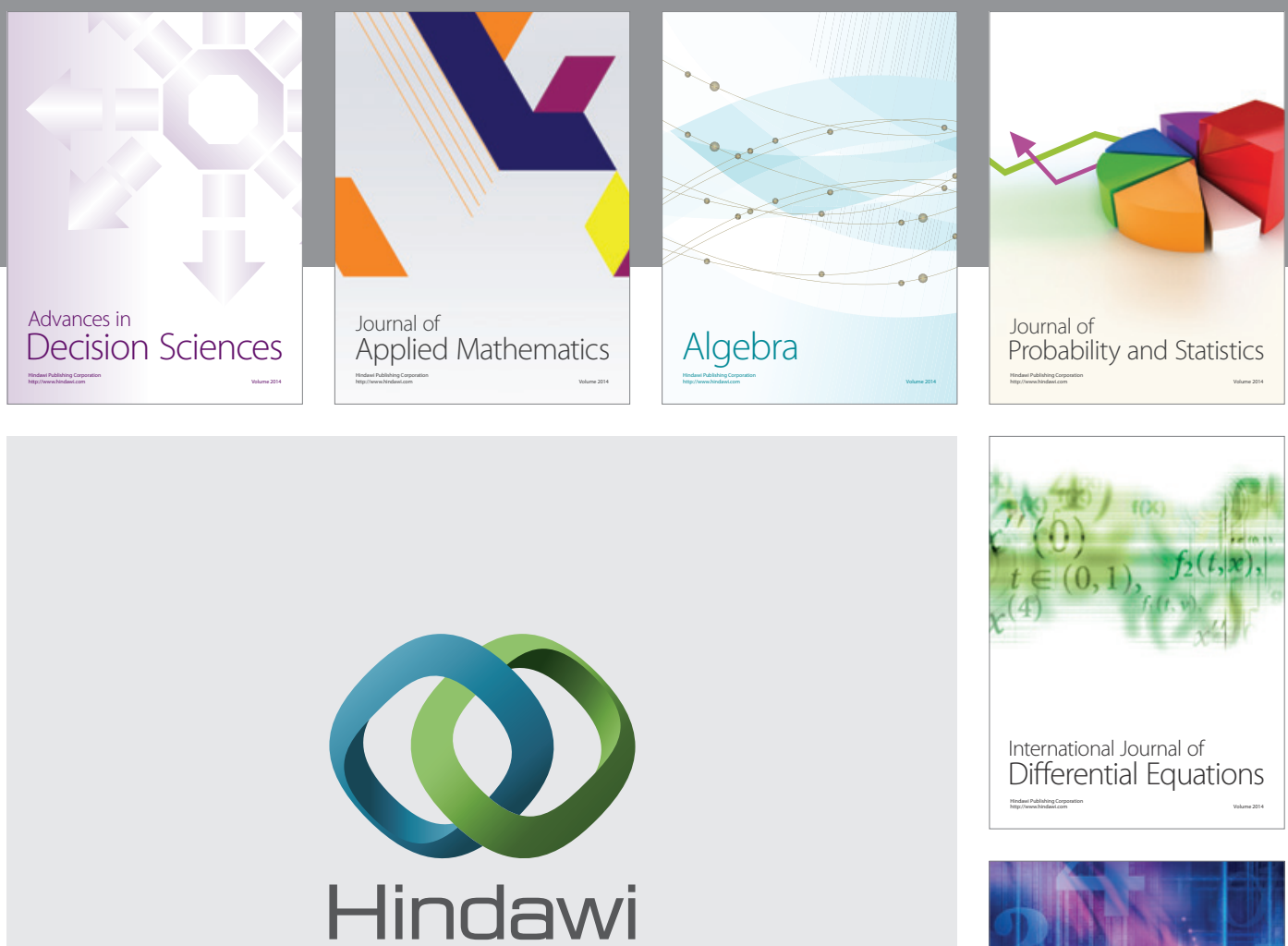

Submit your manuscripts at http://www.hindawi.com
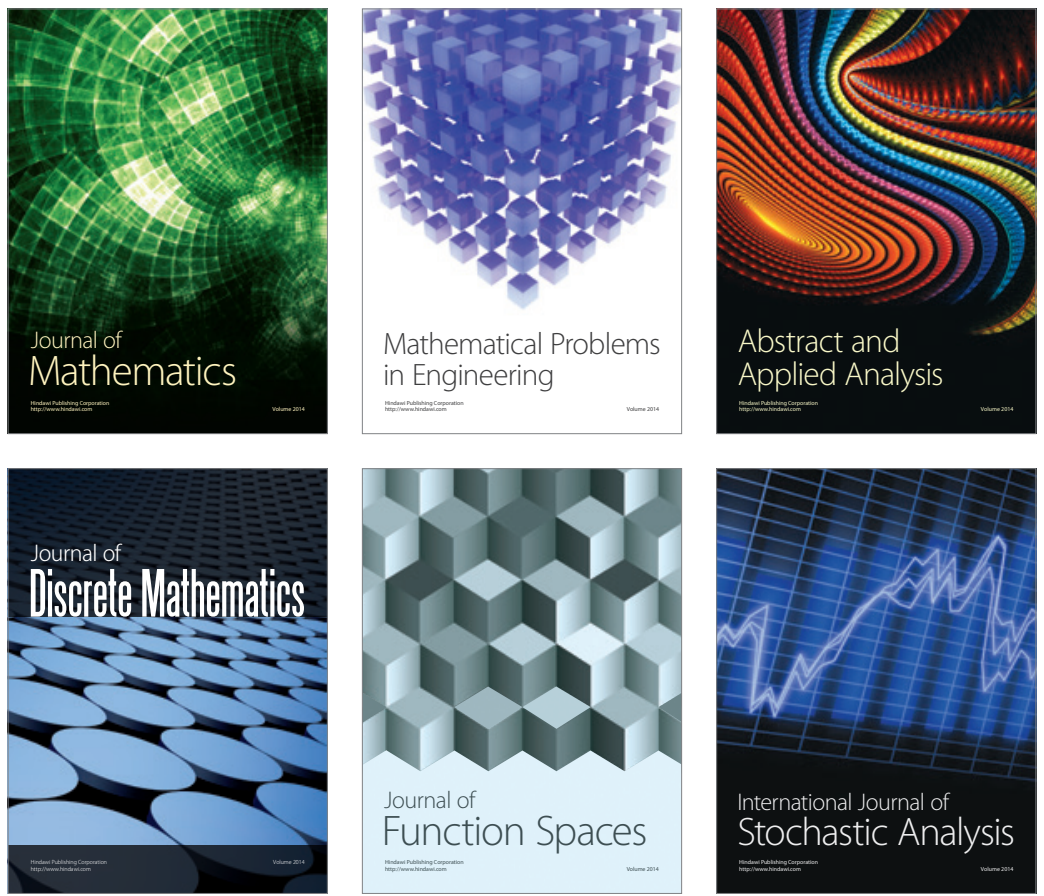

Journal of

Function Spaces

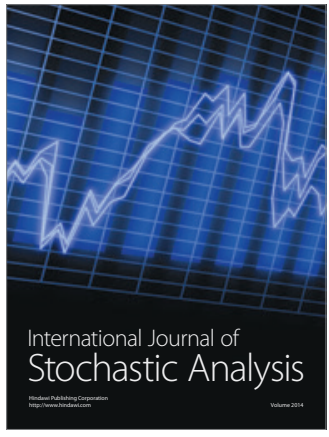

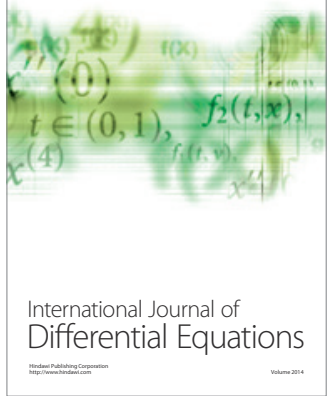
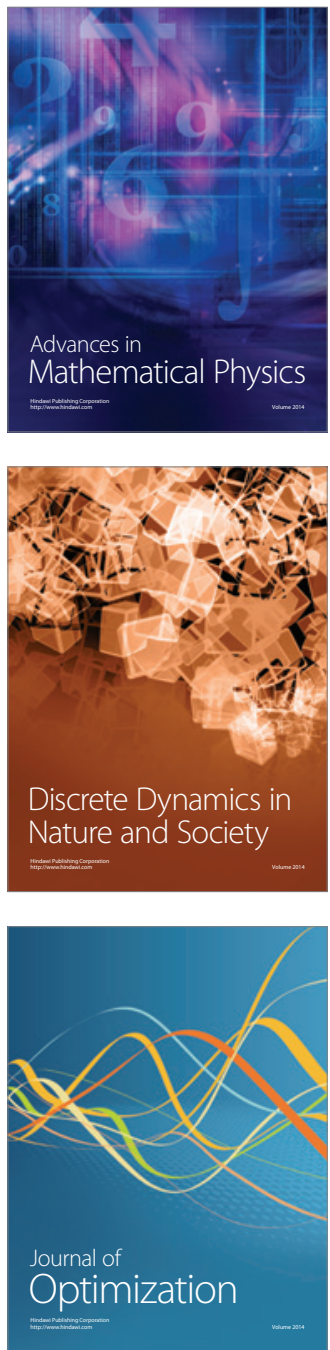